Nach seinem Ausscheiden aus der Politik zog es Maier wieder zurück in die Wissenschaft; 1988 übernahm er den Guardini-Lehrstuhl für christliche Weltanschauung, Religions- und Kulturtheorie an der Universität München. Um die Denomination der Professur, vorher eine für „Religionsphilosophie“, hatte es - typisch Universität - kleinlichen Streit gegeben. Trotz des holprigen Starts wurden die zwölf Jahre bis 1999 eine produktive Zeit: Maier forschte über die Geschichte der christlichen Zeitrechnung und über die modernen Gewaltregime des 20. Jahrhunderts. Vor allem seine Studien über „Totalitarismus und politische Religionen“ bereicherten die Totalitarismusforschung in Deutschland. ${ }^{5}$ Einen großen Dienst für die mit dem Staat befassten Wissenschaften vollbrachte Maier in Zusammenarbeit mit dem Frankfurter Staatsrechtler Michael Stolleis bei der Herausgabe der 21-bändigen Bibliothek des deutschen Staatsdenkens in den Verlagen C.H. Beck und Insel. Hier wurde eine weite terra incognita der älteren deutschen Staatslehre der Öffentlichkeit wieder zugänglich gemacht.

Ein Leben neigt sich dem Ende zu, das glücklicher kaum hätte verlaufen können. Zu diesem Leben gehören auch eine Ehefrau über 50 Jahre, sechs Töchter, neun Enkel und zwei Urenkel (Stand: Juni 2013). Mit seinem Privatleben geht Maier nicht hausieren, er verschweigt es aber auch nicht. Aus den Schilderungen des Erzählers wie auch aus den Notizen der Töchter (vgl. S. 304 - 311) geht das Bild eines warmherzigen, dem Leben zugewandten, immer sich verantwortlich fühlenden Familienmenschen hervor, der sich auch seiner unvermeidlichen Versäumnisse in der Rolle des Erziehers bewusst ist. Seine abschließenden Bemerkungen über das Alter lassen einen sympathischen Charakter hervortreten, der dankbar ist für das Glück, das ihm zuteil wurde - und der gelassen und voller Demut das Unvermeidliche akzeptiert. Dazu gehört auch, dass die Erinnerungen zunehmend wichtiger werden gegenüber dem neu Erlebten. Wohl dem, der wie Maier viele Begabungen hatte, sie sehr weitgehend zu entfalten wusste und (demzufolge) sehr viel Positives zu erinnern hat. Das Buch sei jedem wärmstens ans Herz gelegt, dem Buchliebhaber auch die bereits 2011 erschienene, nur zehn Euro teurere gebundene Version.

Patrick Horst

5 Vgl. Hans Maier, Politische Religionen. Gesammelte Schriften, Band II, München 2007.

\title{
Porträts deutscher Politikwissenschaftler: Standardwerk zu Biographien und Entwicklung des Faches
}

Jesse, Eckhard und Sebastian Liebold (Hrsg.): Deutsche Politikwissenschaftler - Werk und Wirkung. Von Abendroth bis Zellentin, Nomos Verlagsgesellschaft, Baden-Baden 2014, 849 Seiten, $€ 98,-$.

Der Literaturkanon zur deutschen Politikwissenschaft ist „immens“ (S. 11). „Das Fehlen eines Bandes über die führenden Politikwissenschaftler Deutschlands [...] ist gleichwohl augenfällig [...]" (S. 17). Den Herausgebern Eckhard Jesse und Sebastian Liebold gelingt es nahezu meisterhaft, diese Lücke zu schließen. Das vorliegende, in jeder Hinsicht gewichtige 
Werk, porträtiert 50 Politikwissenschaftler und kann somit auch als Geschichte des Faches gelesen werden.

Es stand den Herausgebern frei, aus einer großen Bandbreite an Fachvertretern zu schöpfen, um einige für detaillierte Portraits auszuwählen. Wohl jeder Leser wird an bestimmte Namen denken, die ebenfalls hätten gewürdigt werden sollen, „aber bei einer Anzahl von 50 Personen ist unvermeidlich Dissens präjudiziert" (S. 18). Die Auswahl, die unvermeidbar gewisse Angriffsflächen bietet, kann dennoch als äußerst gelungen und ausgewogen bezeichnet werden. Jesse und Liebold legten vier Kriterien bei der Personenauswahl an: Neben der fachlichen Kompetenz zählt ein erfolgreiches Wissenschaftsmanagement und die Bildung einer Schule. Ferner kommt es auf die öffentliche Sichtbarkeit an. Schließlich stehen solche Wissenschaftler zur Auswahl, die 70 Jahre oder älter sind. Zudem legen die Herausgeber Wert darauf, „dass die Beiträge ein einheitliches 'Gesicht' erhalten und ihre Gliederung zwecks eines besseren Vergleichs möglichst nicht voneinander abweicht" (S. 9). Jedes Kapitel trägt die Überschriften: Vita - Forschungsschwerpunkte - Schulgründung und Wissenschaftsmanagement - Kritische Würdigung - Rezeption.

Nach dieser Vorstellung der Struktur des Buches folgen etwa knapp 20 Seiten über die Wichtigkeit und Bekanntheit (illustriert anhand von Rankings und Festschriften) von Politikwissenschaftlern - ein wenig ertragreicher und ermüdender Abschnitt. Im weiteren Verlauf der Einleitung wird das Wirken der ausgewählten Wissenschaftler anhand thematischer Schwerpunkte kursorisch vorgestellt. Dieses Vorgehen nimmt in vielen Fällen die Inhalte vorweg, die später in den Einzelporträts ausführlich dargestellt werden. Eine kürzere Einleitung (sie umfasst beinahe 80 Seiten) hätte dem Werk insgesamt gut getan.

Für die Herausgeber war es reizvoll, „das Fach im Spiegel seiner Repräsentanten auszuleuchten“ (S. 17). Es sind zum einen die Wissenschaftler selbst, die Erscheinungsbild und Stellenwert einer Disziplin prägen. Zum anderen sind es politische Ereignisse, mithin der Untersuchungsgegenstand, der die Disziplin und ihre wissenschaftliche Bedeutung beeinflusst. Wie sehr die Repräsentanten der Politikwissenschaft und politisch-gesellschaftliche Zeitläufe miteinander verbunden sind, spiegeln markante Begriffe. Sie sind zu Kurzformeln geworden, die das Konstitutive des politischen Koordinatensystems benennen. Karl Dietrich Bracher klassifizierte 1976 - noch unter den Auspizien deutscher Zweistaatlichkeit die Bundesrepublik als eine „postnationale Demokratie unter Nationalstaaten“ (S. 152). Damals ging es ihm um die „Selbstanerkennung der Bundesrepublik“ (S. 153), doch gewiss lässt sich der Begriff im vereinten Europa auch heute verwenden. In ihrem herausragenden Spätwerk mit dem Titel „Deutsche Außenpolitik zwischen Selbstbeschränkung und Selbstbehauptung“ (2001) zeigte Helga Haftendorn, wie die deutsche Außenpolitik der Nachkriegszeit „durch taktisches Geschick und große Beharrlichkeit ihre Ziele verfolgte“ (S. 293). In Haftendorns klassischer und vielfach gebrauchter Formulierung handelte es sich um die „Methode des Souveränitätsgewinns durch Souveränitätsverzicht“ (S. 293). Im Zentrum seines wissenschaftlichen Werkes stand für Dolf Sternberger „die nachhaltige Bejahung der Verfassungsordnung durch die Bürger“ (S. 743). Anlässlich des 30. Gründungstages der Bundesrepublik sprach Sternberger von „Verfassungspatriotismus“, ein Ausdruck, „der seitdem zu einem Leitbegriff der politischen Selbstverständigung in Deutschland geworden ist" (S. 743).

Während die Gründung der deutschen Politikwissenschaft nach dem Krieg noch von „Demokratie-Lernen“ (S. 45) nach US-amerikanischem Vorbild bestimmt war, hat sich das Fach in den nachfolgenden Jahrzehnten etabliert und konsolidiert. Die politischen Um- 
bruchzeiten 1989/90 bedeuteten auch für die Politikwissenschaft einen Aufbruch in neue Themengebiete. Neben bewährte Fragen traten die Transformationsforschung ehemaliger Staaten des Ostblocks, die Analyse der Vereinigungsfolgen und - als Ausdruck von Erweiterung und Vertiefung - neue Aspekte der Europäischen Union. Mit Blick auf zukünftige Schwerpunkte zeigen sich Jesse und Liebold sicher: „Der Staat und die verschiedenen Herrschaftsformen bleiben ein zentrales, wenn nicht das zentrale Forschungsfeld der Politikwissenschaft (Governance) [...]“ (S. 67). Naturgemäß unterliegt jede Forschungsagenda immer Moden und Themenkonjunkturen. Auch sie sind es, die den Fortschritt einer Disziplin beschleunigen können. Die aktuelle Ortsbestimmung der Politikwissenschaft durch Jesse und Liebold, wonach es „heute nicht en vogue“ sei, das Fach „als praktische Wissenschaft zu betreiben“ und es „gar als 'praktische Philosophie' zu verstehen, reichlich unzeitgemäß“ (S. 66) sei, sind wenig überzeugend. Anregender ist hingegen ihr Rat an die Policy-Forschung, „perspektivisch eher regionale Vergleiche“ (S. 68) anzustellen (wie zum Beispiel über Arbeitsbedingungen, Freizeitverhalten, Ehrenamt).

Angesichts seines Umfanges und seiner programmatischen Anlage ist das vorliegende Buch in erster Linie ein Nachschlagewerk. Gleichzeitig bietet es einen hohen Lesegenuss. Besonders interessant wird die Lektüre, wenn sich Biografisches mit zeitgeschichtlichen Schilderungen verbindet. Über Winfried Steffanis Wirken stellt Patrick Horst fest, dass Steffani im damaligen Zeitgeist keinen Verbündeten fand; „wackere Konservative wie er standen in den 1970er Jahren in den Sozialwissenschaften auf weitgehend verlorenem Posten - zumal in einer sozialdemokratischen Hochburg wie Hamburg “ (S. 734). Die Kontinuität im wissenschaftspolitischen Klima bis zum heutigen Tag kann hier wohl als bemerkenswert gelten. Als in den 1990er Jahren die mangelnde Leistungsfähigkeit des politisch-administrativen Systems thematisiert und auch die Politikwissenschaft vielfach in die Klagegesänge des „Reformstaus“ einstimmte, griff Fritz W. Scharpf auf sein Konzept der „Politikverflechtungsfalle“ zurück und sprach von der „gefesselten Republik“. In der Summe, so resümiert Manfred G. Schmidt, „waren die Reformen in Deutschland zahlreicher, als es Scharpfs Analysen der deutschen Politik erwarten lassen [...]“ (S. 650). Kurz gesagt: „Scharpf unterschätzt bisweilen die Beweglichkeit der deutschen Politik“ (S. 650). Die Intelligenz des arbeitenden Staates und die Stärke exekutiver Führerschaft ließen die seinerzeitige Beschreibung vom Reformstau als überdehntes Zerrbild erscheinen. So sah es auch Gerhard Lehmbruch. Zu der Forderung von Bundespräsident Roman Herzog, durch das Land müsse ein „Ruck“ gehen, schrieb Lehmbruch: „So etwas mag anlässlich einer Hoteleröffnung gewisse wechselseitige Aufmerksamkeitseffekte bringen, ersetzt aber keine institutionelle Analyse" (S. 490).

Neben fachlichen Aspekten erfährt der Leser auch etwas über die Eigentümlichkeiten der Porträtierten. Klaus von Beyme wird charakterisiert als „einer der produktivsten deutschen Politikwissenschaftler, wenn nicht sogar derjenige mit dem umfangreichsten Oeuvre überhaupt“ (S. 116). National organisierte Staatlichkeit sowie auch Regierungsinstitutionen jenseits staatlicher Einhegung stehen im Zentrum des Interesses bei Beate Kohler-Koch. Auf Publikationen an prominenter Stelle hat sie nie besonders großen Wert gelegt, „sondern ganz im alten Stil darauf vertraut, dass sich gute Argumente schon durchsetzen werden“ (S. 459). Für Eckhard Jesse ist Hans-Peter Schwarz ein „glänzender Stilist“, dessen „Sottisen“, bisweilen aus einer „flotten Feder" kommen (S. 693).

Alle Porträtierten haben die deutsche Politikwissenschaft geprägt, haben in Form von Promovenden und Habilitanden Schüler hervorgebracht und vielfach auch als Ratgeber in 
die praktische Politik hineingewirkt. Auffällig ist jedoch die immer wiederkehrende Feststellung, dass von einer richtigen Schulbildung nicht die Rede sein könne. Die als „Freiburger" „Heidelberger“ und „Marburger Schule“ bezeichneten wissenschaftlichen Denkzweige sind bekannt, doch eine „Berliner“ Schule hat sich nicht herausbilden können, „schon wegen des Fehlens eines Gravitationszentrums“ (S. 13).

Den Herausgebern ist die Komposition eines durchaus als monumental zu bezeichnenden Bandes gelungen, der sich gewiss als Standardwerk behaupten wird. Der Gesamtertrag der Porträts ist hoch. In der Verdichtung von Namen, politischen Entwicklungen der Bundesrepublik, Entstehungsspuren des Faches sowie von Forschungsgegenständen wirken die Kapitel mitunter wie ein Pumpwerk der Politikwissenschaft. Hier liegt ein biografisches Handwörterbuch vor, das auch Auskunft über die Entwicklung des Faches selbst gibt. Dieses Buch ist wie Goldstaub für jede Bibliothek eines politikwissenschaftlichen Seminars.

Helge F. Jani

Politische Biographien: empfehlenswerte Sammlung für die politische Bildung

Rahmig, Jürgen: Politiker mit Leib und Seele, Oertel + Spörer Verlag, Reutlingen 2013, 292 Seiten mit Abbildungen, € 19,90.

Die Absicht des Autors ist lobenswert: Ausgehend von dem verbreiteten Vorurteil, dass sich immer weniger junge Menschen für Politik interessieren, hat sich Jürgen Rahmig vorgenommen, „Lust auf Politik“ (Rückseitentext) zu machen. Die Prämisse, von der er ausgeht, ist auch richtig, wie einschlägige Untersuchungen zur Politikverdrossenheit zeigen: Jugendliche sind nicht generell an Politik desinteressiert - nur für Parteipolitik können sie sich nicht erwärmen. Sie wollen sich politisch beteiligen, aber vorzugsweise an den so genannten „unkonventionellen“ Formen der Politik. Die Idee des Politikredakteurs beim Reutlinger General-Anzeiger hat Charme: Indem er 19 prominente Politiker Baden-Württembergs in Kurzporträts vorstellt, sie über ihren Weg in die Politik, ihre (politischen) Leidenschaften und Abneigungen erzählen lässt, möchte er junge Menschen auch für die „konventionelle“ Parteipolitik begeistern, die derzeit nicht in besonders hohem Kurs steht. Sie sollen erkennen, dass auch Politiker „Menschen sind wie du und ich, mit ihren Schwächen und Stärken, ihren Vorlieben und Antipathien“ (Klappentext).

Nicht zufällig macht mit der grünen Bundestagsabgeordneten Agnieszka Brugger die jüngste der vorgestellten Politikerinnen den Anfang: Sie zog bereits 2009 mit 24 Jahren erstmals in den Bundestag ein, damals noch unter ihrem Mädchennamen Malczak. Agnieszka Brugger hat Politikwissenschaft in Heidelberg studiert, sie trägt Piercings an Nase und Unterlippe, ist insofern durchaus eine unkonventionelle Abgeordnete. Von Menschen im Wahlkampf darauf angesprochen, entgegnete sie: „(B)ei einer Politikerin ist wichtig, was aus dem Mund rauskommt und nicht, was am Mund dran ist“ (S. 14). Die Bundestagsabgeordnete entspricht auch sonst nicht typischen Klischees: Sie ist gebürtige Polin (ihre Eltern waren Spätaussiedler), Vegetarierin - was für Grüne so ungewöhnlich aber auch nicht ist - und war in der Jugend als einzige Frau Mitglied in einem Boxverein. Bemerkenswert 